\title{
Spontaneous necrosis of parathyroid adenoma: biochemical and imaging follow-up for two years
}

\author{
W B Chan, C C Chow, A D King, V T F Yeung, J K Y Li, W Y So, C S Cockram
}

\begin{abstract}
Summary
We report a patient with a biochemically and radiologically confirmed parathyroid adenoma, which underwent spontaneous resolution by necrosis. The patient was followed-up over the subsequent two years during which time the tumour and hypercalcaemia recurred. Sequential radiological and biochemical changes at the time of diagnosis, spontaneous necrosis and recurrence are documented fully.
\end{abstract}

Keywords: hyperparathyroidism; hypocalcaemia; apoplexy

Primary hyperparathyroidism is one of the commonest endocrine disorders. However, spontaneous resolution by necrosis of a parathyroid adenoma is rare. Although there have been approximately 10 reported cases since the condition was first described in $1946,{ }^{1}$ in most cases the infarcted gland was removed soon after diagnosis. Therefore, the natural history, biochemical and hormonal changes following spontaneous infarction are not well known. We report a patient who had spontaneous infarction of her parathyroid adenoma, while awaiting surgery, with detailed documentation of the above-mentioned features.

\section{Department of \\ Medicine and \\ Therapeutics, Prince \\ of Wales Hospital, The \\ Chinese University of \\ Hong Kong, Hong \\ Kong \\ W B Chan \\ C C Chow \\ J K Y Li \\ W Y So \\ C S Cockram}

\section{Department of}

Radiology and Organ

Imaging, Prince of

Wales Hospital, The

Chinese University of

Hong Kong, Hong

Kong

A D King

\section{Department of}

Medicine, Our Lady of

Maryknoll Hospital,

Hong Kong.

V T F Yeung

Submitted 1 June 1999

Accepted 13 August 1999

\section{Case report}

A 78-year-old woman was referred to our clinic for further management of Type 2 diabetes. She gave a history of renal stone, with right nephrectomy, 8 years before. She was put on oral hypoglycaemic therapy. She was found incidentally to have hypercalcaemia with a calcium concentration of $3.43 \mathrm{mmol} / 1$ (normal range $2.20-2.62 \mathrm{mmol} / \mathrm{l}$ ) together with a reduced phosphate concentration of 0.55 $\mathrm{mmol} / \mathrm{l}(0.82-1.40 \mathrm{mmol} / \mathrm{l})$. Alkaline phosphatase was normal at 85 IU/1 (45-145 IU/1). There was renal impairment with a plasma creatinine concentration of $160 \mu \mathrm{mol} / 1$ (44-107 $\mu \mathrm{mol} / \mathrm{l})$, but she had stable glycaemic control as indicated by a $\mathrm{HbA}_{1 c}$ concentration of $7.4 \%$. Physical examination revealed mild peripheral neuropathy and the nephrectomy scar, but no obvious neck mass. Further investigations confirmed primary hyperparathyroidism. Her parathyroid hormone (PTH) was $44 \mathrm{pmol} / 1$ (1.16-5.67 pmol/1, intact PTH assay; coefficient of variation $7 \%$ ) with concomitant plasma calcium of $3.40 \mathrm{mmol} / 1$ and ionized calcium of $1.61 \mathrm{mmol} / 1(1.13-1.32 \mathrm{mmol} / \mathrm{l})$.
Ultrasound of the abdomen confirmed the previous right nephrectomy but showed no other abnormality. Her hypercalcaemia improved to approximately $2.8 \mathrm{mmol} / 1$ with adequate hydration, a low calcium diet and oral phosphate solution. She was referred for parathyroidectomy and was on the waiting list for surgery. During subsequent follow-up, her alkaline phosphatase gradually increased to $1465 \mathrm{IU} / 1$, indicating development of parathyroid bone disease. Her glycaemic control was stable on oral hypoglycaemic therapy and there was no further deterioration of diabetic complications.

She was put on the waiting list for surgery, which was very long in our hospital. Two years later, she underwent pre-operative imaging to localise the parathyroid adenoma. Computed tomography (CT) showed a $2.5 \times 1.5 \mathrm{~cm}$ welldefined parathyroid adenoma posterior to the upper pole of the left lobe of the thyroid. The adenoma was of homogenous soft tissue attenuation and revealed diffuse enhancement after contrast (figure 1). However, 4 months later, before surgery could be performed, she was admitted to our hospital having sustained a fracture of the right neck of femur after a fall. On admission, she was found to be hypocalcaemic (plasma calcium concentration 1.37 $\mathrm{mmol} / \mathrm{l}$ (albumin adjusted)). Plasma phosphate was $0.89 \mathrm{mmol} / 1$, magnesium was $0.55 \mathrm{mmol} / 1$ (0.67-1.01 mmol/l) and alkaline phosphatase was $1730 \mathrm{IU} / 1$. Trousseau's sign was positive and the electrocardiogram showed a prolonged QT interval. She was treated with intravenous calcium gluconate followed by oral calcium supplementation and calcitriol. Magnesium was replaced with oral magnesium chloride. Two days later, the PTH concentration was $32.5 \mathrm{pmol} / 1$ and the calcium concentration $1.84 \mathrm{mmol} / \mathrm{l}$. Serum 25-OH vitamin D3 concentration was $34 \mathrm{nmol} / 1(10-50 \mathrm{nmol} / \mathrm{l})$. A CT scan of the neck showed no change in the size of the parathyroid adenoma but now there was evidence of central necrosis with a low attenuation centre which failed to enhance after contrast (figure 2). Plasma calcium was maintained within the normal range with calcitriol and oral calcium, and a right hip replacement was performed uneventfully.

On subsequent follow-up, her calcium level was maintained within the normal range and calcium and calcitriol doses were gradually reduced over a period of 8 months. The parathyroid hormone concentration dropped gradually to $5.4 \mathrm{pmol} / 1$ one month after admission and then showed a gradual rise during 


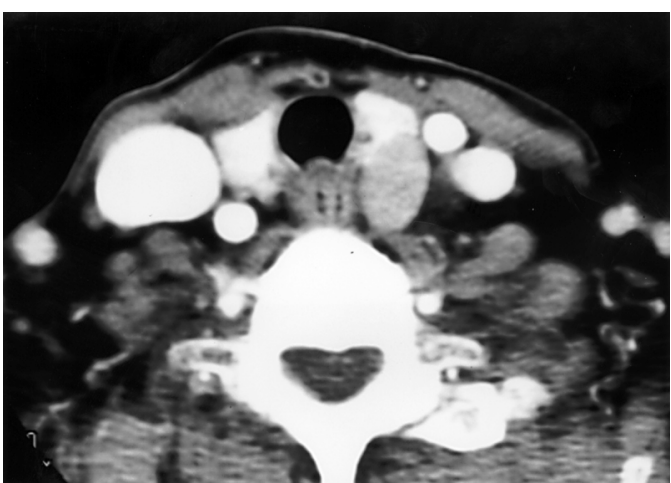

Figure 1 Contrast CT scan of the neck showing a parathyroid adenoma posterior to the left lobe of the thyroid at diagnosis

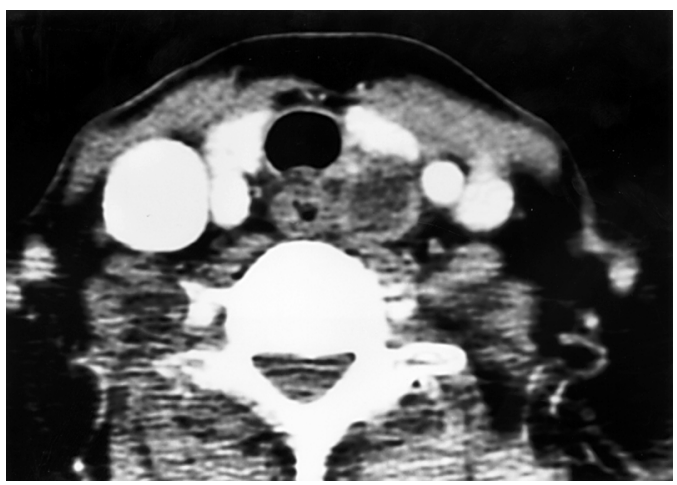

Figure 2 Contrast CT scan of the neck at the time of spontaneous necrosis

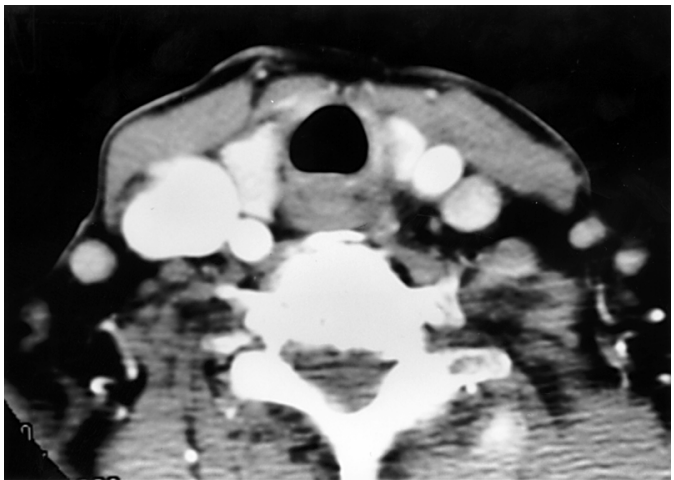

Figure 3 Contrast CT scan of the neck at the time of involution

further follow-up. A further CT scan of the neck 3 months later showed involution of the parathyroid adenoma which had almost disappeared, leaving a residual $5 \mathrm{~mm}$ soft tissue mass (figure 3). Her calcium concentration rose beyond the normal range one month after calcium and calcitriol were stopped. Recurrence of primary hyperparathyroidism was confirmed with concomitant measurements of PTH $(24.2$ $\mathrm{pmol} / \mathrm{l})$ and plasma calcium $(2.68 \mathrm{mmol} / \mathrm{l})$. Details of the biochemical changes and clinical events are summarised in figure 4 .

\section{Discussion}

Parathyroid apoplexy, as described by Eric Nylen to signify parathyroid spontaneous

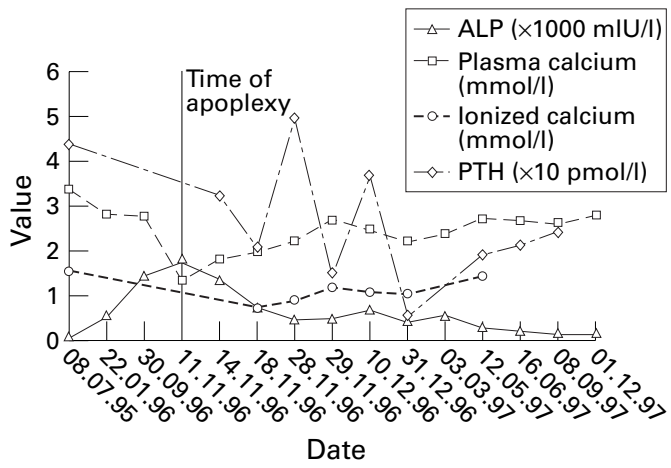

Figure 4 Biochemical profile over 2 years

necrosis or haemorrhage, is a rare complication of primary hyperparathyroidism. ${ }^{2}$ So far, only around 10 cases have been reported in the literature. Its presentation can be quite variable, including pressure symptoms due to haemorrhage, ${ }^{3-6}$ neck pain, ${ }^{7-9}$ hypercalcaemia due to acute release of stored $\mathrm{PTH},{ }^{10}$ hypocalcaemia due to autoparathyroidectomy, ${ }^{12811}$ or normalisation of plasma calcium..$^{9} 1213$ The biochemical changes can be drastic if there is significant necrosis, and death has been reported due to severe hypercalcaemia. ${ }^{10}$ Most reported cases were treated by parathyroidectomy after the event and therefore the longterm consequences and clinical course remain unclear.

As our patient was already 77 years old at the time of parathyroid apoplexy, and was reluctant to undergo any further surgical intervention, it provided us with an opportunity to examine the subsequent course of this condition. She was relatively asymptomatic, despite severe hypocalcaemia. We did not observe a phase of transient worsening of hypercalcaemia. This may be due to delayed presentation, but agrees with some case reports of patients with normal or low calcium concentration at the time of presentation. ${ }^{12811}$ Of those cases in whom PTH concentration had been measured at the time of presentation, most showed a decrease in PTH soon after the episode of necrosis. ${ }^{29}$ However, our patient had a persistently raised PTH level at presentation and subsequently showed a further rise to $49.4 \mathrm{pmol} / 1$. Although the PTH level remained above the normal range, it stayed well below the initial level. We postulate that the abrupt drop in PTH due to parathyroid necrosis resulted in hypocalcaemia and hungry bone syndrome. The situation closely simulates that of surgical parathyroidectomy. This may have further triggered a secondary or compensatory response. This is supported by the observation that the PTH concentration gradually returned to near normal as the hypocalcaemia resolved with treatment. The other possibility was osteomalacia due to vitamin $\mathrm{D}$ deficiency. There has been a reported case of osteomalacia associated with primary hyperparathyroidism ${ }^{14}$; however, the normal vitamin D3 level excluded this diagnosis.

Although the necrotic parathyroid adenoma was not excised and hence there was no histo- 
logical confirmation, the characteristic CT and clinical features appear confirmatory. In addition, the CT scan several months later showed continuous involution of the initial necrotic adenoma. This further confirmed the initial diagnosis of parathyroid adenoma necrosis. There have been reports of primary hyperparathyroidism entering remission after parathyroid gland necrosis. ${ }^{2}{ }^{13} 15$ However, in most reported cases, the necrotic parathyroid adenoma were excised. ${ }^{91315}$ A recent report by Nylen et al showed that the primary hyperparathyroidism could enter remission even without surgical excision. ${ }^{2}$ By contrast, our patient exhibited recurrence of the disease 9 months after the acute event and the repeated CT 3 months after the event still showed a $5-\mathrm{mm}$ soft tissue mass suggesting a remnant adenoma. Thus, such patients need to be followed closely as recurrence with a need for treatment is possible.

The cause of necrosis in our patient remains uncertain. There was no evidence to suggest previous hypotension, angiography ${ }^{16}$ or thyroid surgery, ${ }^{5}$ as suggested by previous reports, although hypotension related to fracture is still a possible cause. However, one common feature shared by all reported cases is that the adenomas exceed $1 \mathrm{~cm}$ in diameter, most

1 Norris EH. Primary hyperparathyroidism. Arch Pathol 1946;42:261.

2 Nylen E, Shah A, Hall J. Spontaneous remission of primary hyperparathyroidism from parathyroid apoplexy. $\mathcal{f}$ Clin

3 Hotes LS, Barzilay J, Cloud LP, Rolla AR. Spontaneous hematoma of a parathyroid adenoma. Am $\mathcal{F}$ Med Sci 989;297:331-3.

4 Berry BE, Carpenter PC, Fulton RE, Danielson GK. Mediastinal hemorrhage from parathyroid adenoma simulating dissecting aneurysm. Arch Surg 1974;108:740-1.

5 Pearl MA, Sternerg WH, Dingman JF. Unusual cases of hyperparathyroidism. Arch Intern Med 1962;110:481.

6 Jordan FT, Harness JK, Thompson NW. Spontaneous cervical hematoma: a rare manifestation of parathyroid adenoma. Surgery 1981;89:697-700.

7 Howard JE, Follis RH, Yendt ER, et al. Hyperparathyroidism. Case report illustrating spontaneous remission due to necrosis of adenoma, and a study of the incidence of necrosis in parathyroid adenoma. F Clin Endocrinol 1953; 13: necrosis in

8 Northcutt RC, Levinson JD, Earnest JB. Hypocalcaemia resulting from infarction of a parathyroid adenoma. Ann resulting from infarction of

9 Mclatchie GR, Morris EW, Forrester A, Fogelman I. Autoparathyroidectomy: a case report. Br f Surg 1979;66: $552-3$.

\section{Learning points}

- primary hyperparathyroid patients with parathyroid adenoma greater than $1 \mathrm{~cm}$ diameter need early surgical excision in order to avoid potential crisis of apoplexy

- surgical removal of adenoma is still indicated after apoplexy due to the possibility of recurrence

exceeding $2 \mathrm{~cm}$. Relatively large size may increase the risk of ischaemia and necrosis, and this may be an additional indication for early surgical intervention in subjects with parathyroid adenomas exceeding $1 \mathrm{~cm}$ in size.

\section{Conclusion}

In summary, we present a case of parathyroid apoplexy complicated initially by hypocalcaemia, but with later recurrence of hypercalcaemia. The later recurrence of disease weighs in favour of surgical intervention in these patients. Review of the literature indicates that this complication occurs primarily in subjects with large parathyroid adenomas $(>1 \mathrm{~cm}$ diameter).

10 Dowlatabadi H. Acute fatal parathyroid poisoning associated with necrosis of the parathyroid adenoma prior to death. F Clin Endocrinol 1959;19:1481.

11 Johnston CC, Schnute RB. A case of primary parathyroidism with spontaneous remission following infarction of the adenoma with development of hypocalcemia tetany. $\mathcal{F}$ Clin Endocrinol 1961;21:196.

12 Connor TB, Clark JW, Martin LG, Lovice H. Intermittent hyperparathyroidism. Trans Am Clin Climatol Assoc 1965;77: 80.

13 Mir R, Gerold T, Khan S, Weitz J. Spontaneous infarction of parathyroid adenoma: a case report and literature review. Head Neck 1993;15:566-8.

14 Natsui K, Tanaka K, Suda M, et al. Spontaneous remission of primary hyperparathyroidism due to hemorrhagic infarction in the parathyroid. Intern Med 1996;35:646-9.

15 Li JKY, Chow CC, Yeung VTF, et al. Primary hyperparathyroidism complicated by osteomalacia. Ann Clin Biochem 1996;33:571-4.

16 Farndon JR, Dale JK, Welles SA Jr. Selective arteriography causing infarction of a parathyroid adenoma. $f \mathrm{R}$ Soc Med 1982;75:978-80. 


\title{
Acute myoedema: an unusual presenting manifestation of hypothyroid myopathy
}

\author{
A Bhansali, V Chandran, J Ramesh, A Kashyap, R J Dash
}

\begin{abstract}
Summary
We describe a patient with primary hypothyroidism due to autoimmune thyroiditis, presenting with acute myoedema and spontaneous rhabdomyolysis. During his hospital stay, he developed altered sensorium due to hypo-osmolal hyponatraemia and later developed bilateral foot drop that responded to appropriate treatment.
\end{abstract}

Keywords: hypothyroidism; myoedema; rhabdomyolysis

Muscle weakness, aches and cramps, stiffness and delayed tendon jerk relaxation are usual features of hypothyroid myopathy (30-80\%), while muscle hypertrophy, myoedema and wasting are occasionally seen. ${ }^{12}$ They evolve gradually over a long period of time. We report a patient with primary hypothyroidism, who presented with acute generalised myoedema, stiffness and spontaneous rhabdomyolysis.

\section{Case report}

A 41-year-old man presented with generalised body swelling and increasing stiffness for 2 weeks. He had experienced severe aches and pains, lower limb weakness and oliguria, for 3 days prior to his admission. He denied any form of strenuous muscle exercise. $\mathrm{He}$ had been a diagnosed hypertensive for 8 years with poor drug compliance. He was diagnosed to have autoimmune thyroiditis with primary hypothyroidism 6 years ago, requiring thyroxine for 6 months only. He remained euthyroid (serum thyroid-stimulating hormone (TSH) $2.5 \mu \mathrm{IU} / \mathrm{ml}$ ) thereafter until about a month prior to admission.

On examination, he was pale, afebrile but tachypnoeic, had peri-orbital puffiness and no goitre. His pulse was 78 beats/min regular, blood pressure $170 / 110 \mathrm{mmHg}$ and voice was hoarse. All his limb muscles were swollen, firm, nonpitting, severely stiff and hypertonic but distinctly nontender, characteristically described as myoedema. He had proximal muscle weakness in both shoulder (4/5) and pelvic girdles $(3 / 5)$ and had pseudomyotonia. Relaxation of deep tendon reflexes was grossly delayed. He had a few coarse crepitations in the left infrascapular region. Other systemic examinations were normal.

Investigations revealed: haemoglobin 10.7 $\mathrm{g} / \mathrm{dl}$, total leucocyte count $10 \times 10^{9} / 1$ with $90 \%$ polymorphs, serum $\mathrm{Na}^{+} 125 \mathrm{mEq} / \mathrm{l}, \mathrm{K}^{+} 3.6$ $\mathrm{mEq} / \mathrm{l}$, urea $14.2 \mathrm{mmol} / \mathrm{l}$, creatinine 133 $\mu \mathrm{mol} / 1$, random blood glucose $6 \mathrm{mmol} / 1$, serum aspartate transaminase $19 \mathrm{IU} / 1$, alanine transaminase $77 \mathrm{IU} / 1$, creatine kinase-MM (CK-MM) $80730 \mathrm{IU} / 1$ (normal up to $20 \mathrm{IU} / \mathrm{l}$ ) and aldolase $35 \mathrm{IU} / 1\left(1-7.6\right.$ at $\left.37^{\circ} \mathrm{C}\right)$ and serum myoglobin $>85 \mathrm{ng} / \mathrm{ml}$. The centrifuged plasma was normal in colour. Arterial blood gas analysis showed $\mathrm{PaO}_{2} 83, \mathrm{PaCO}_{2} 30, \mathrm{pH}$ 7.5, $\mathrm{HCO}_{3}$ 23.9. His serum T3 was $0.2 \mathrm{ng} / \mathrm{ml}$ (0.6-1.2), T4 $9 \mathrm{ng} / \mathrm{ml}$ (60-130), TSH 58.9 $\mu \mathrm{IU} / \mathrm{ml}(0.3-5)$, cortisol at $08.00 \mathrm{~h} 500 \mathrm{nmol} / 1$ (400-600) and at $16.00420 \mathrm{nmol} / 1$ (200-400). Antimicrosomal antibody titre was abnormally elevated. Electromyogram (EMG) revealed polyphasic action potentials consistent with myopathy.

$\mathrm{He}$ received intravenous dextrose-saline, rapidly increasing doses of L-thyroxine from a starting dose of $25 \mu \mathrm{g}$ to $150 \mu \mathrm{g}$ in one week, cefotaxime and amikacin. On day 3 of admission, he became drowsy and disoriented, when his serum $\mathrm{Na}^{+}$was $110 \mathrm{mEq} / \mathrm{l}$, serum osmolality $232 \mathrm{mOsmol} / \mathrm{kg}$ and urine osmolality $345 \mathrm{mOsmol} / \mathrm{kg}$, indicating hypo-osmolal hyponatraemia. In addition to intravenous saline, he received oral salt supplementation and intravenous hydrocortisone. His sensorium gradually improved over the following $10-12 \mathrm{~h}$ with an increase in serum $\mathrm{Na}^{+}$to 124 $\mathrm{mEq} / \mathrm{l}$ and serum osmolality to $258 \mathrm{mOsmol} /$ $\mathrm{kg}$. Urine spot $\mathrm{Na}^{+}$dropped from $61 \mathrm{mEq} / 1$ to $30 \mathrm{mEq} / 1$. On day 5, he developed bilateral foot drop with sensory loss in L4-5/S1 dermatomes. Nerve conduction velocity showed impaired conduction through the common peroneal nerve. After 3 months of thyroxine replacement, his muscle rigidity and stiffness were markedly reduced and power in foot dorsiflexors improved to 3/5. At 6 months of follow-up serum CK-MM had decreased to $432 \mathrm{IU} / 1$ and serum myoglobin was undetectable.

\section{Discussion}

The clinical spectrum of hypothyroid myopathy is varied. Delayed relaxation of tendon jerks and proximal muscle weakness correlate with biochemical severity of hypothyroidism (serum $\mathrm{T} 4<20 \mathrm{ng} / \mathrm{ml}$ ). ${ }^{3}$ Acute myoedema and rhabdomyolysis are, however, distinctly rare. ${ }^{24}$ Raised serum CK-MM and myoglobin, typical myopathic EMG, low T4 and high TSH, and their reversibility with L-thyroxine treatment supported the diagnosis of hypothyroid myopathy with rhabdomyolysis. 
Transient hypothyroidism many years earlier followed by complete clinical and biochemical recovery suggested autoimmune thyroiditis, which eventually led to atrophy of the thyroid and hypothyroidism. The muscle involvement in hypothyroidism is caused by changes in muscle fibres from fast twitching type II to slow twitching type I fibres, deposition of glycosaminoglycans, poor contractility of actin-myosin units, low myosin ATPase activity and low ATP turnover in skeletal muscle. ${ }^{5}$

Hypo-osmolal hyponatraemia is occasionally encountered in primary hypothyroidism. It is accompanied by a decrease in glomerular filtration rate, inappropriately elevated circulating antidiuretic hormone, because of its slow catabolism, and concomitant cortisol deficiency. ${ }^{6}$ Bilateral symmetrical foot drop in

1 Ramsey I. Muscular abnormalities of hypothyroidism. In Thyroid disease and muscle dysfunction. London: William Heinemann, 1974; pp 126-171.

2 McArdle B. Metabolic and endocrine myopathies. In: Walton JN, ed, Disorders of voluntary muscle. Edinburgh: Walton JN, ed, Disorders of volunt

3 Khaleeli AA, Griffith DG, Edwards RHT. The clinical presentation of hypothyroid myopathy and its relationship to abnormalities in structure and function of skeletal muscle. Clin Endocrinol 1983;19:365-76.

4 Lochmuller H, Reimers CD, Fischer P, Heuss D, MullerHocker J, Pongratz DE. Exercise induced myalgia in hypothyroidism. Clin Invest 1993;71:999-1001.

\section{Learning points}

- myoedema, muscle hypertrophy, and wasting are uncommon features of hypothyroid myopathy

- CK-MM can be used as a marker of hypothyroid myopathy

- hypothyroid myopathy responds to L-thyroxine therapy

- hypo-osmolal hyponatraemia can be an important cause of altered sensorium in long-standing untreated hypothyroidism

this patient was possibly caused by peroneal neuropathy due to decubitus pressure or glycosaminoglycans deposition in the perineural sheath. ${ }^{7}$

5 Wiles CM, Young A, Jones DA, Edwards RHT. Muscle relaxation rate, fibre type composition and energy turnover in hyper and hypothyroid patients. Clin Sci 1979;57:375-84.

6 Macaron C, Famuyiwa O. Hyponatremia of hypothyroidism; appropriate suppression of antidiuretic hormone levels. Arch Intern Med 1978;138:820-3.

7 Swanson JW, Kelly JJ Jr, McConahey WM. Neurologic aspects of thyroid dysfunction. Mayo Clin Proc 1981;56: 504-12.

\title{
Hepatocellular adenomatosis associated with hereditary haemochromatosis
}

\author{
J M Radhi, J Loewy
}

\begin{abstract}
Summary
A young healthy man presented with abdominal pain following an accidental fall. Imaging studies and laparoscopy revealed multiple yellowish well-defined hepatic lesions. Liver biopsies showed hepatic adenomas and iron overload. Laboratory investigation confirmed a diagnosis of hereditary haemochromatosis. To our knowledge this represents the first report of an association of hepatic adenomatosis and primary haemochromatosis.
\end{abstract}

University of

Saskatchewan, Royal

University Hospital,

103 Hospital Drive,

Saskatoon,

Saskatchewan

S7N 0W8, Canada

Department of

Pathology

J M Radhi

Department of

Medical Imaging

J Loewy

Submitted 21 April 1999 Accepted 13 August 1999
Hereditary haemochromatosis is known to be complicated by hepatic cirrhosis and carcinoma but not adenomas.

\section{Case report}

A 26-year-old man presented to the emergency room 7 years ago with abdominal pain following an accidental fall. On examination, he was found to have tenderness throughout the right upper quadrant and a palpable lower liver edge. A presumptive diagnosis of liver injury was made and the patient was admitted for observation. A computed tomography (CT) scan at the time demonstrated multiple intrahepatic lesions of variable sizes ranging from less than $1 \mathrm{~cm}$ to $5 \mathrm{~cm}$, with no suggestion of intra-abdominal haemorrhage or injury (figure 1). The differential diagnosis based on the CT scan included multiple haemangiomas and the possibility of metastatic disease. In view of the liver function tests, carcinoembryonic antigen and alpha-feto protein markers were assayed and were within normal range. A liver biopsy was performed and revealed hepatic adenoma with parenchymal iron overload.

The patient's condition stabilised and he was discharged to annual follow-up by clinical and radiological examination. He presented again in 1995 with abdominal discomfort. Labora- 


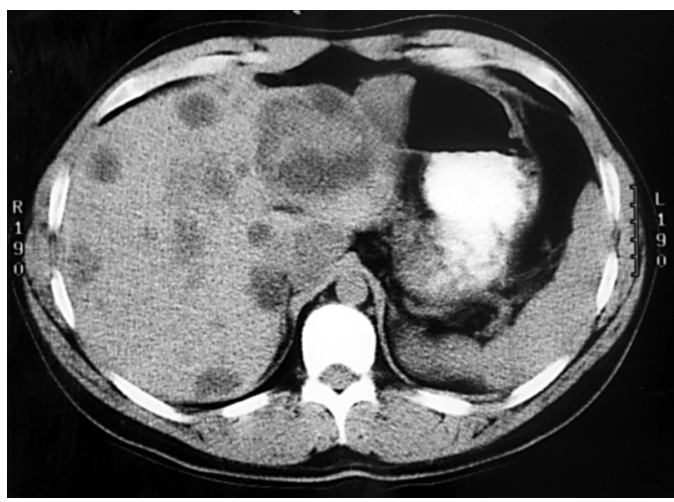

Figure 1 Enhanced CT scan taken at the level of the splenic artery shows multiple low attenuation lesions. The largest lesion anteriorly measures $5.0 \mathrm{~cm}$ and shows inhomogeneity

tory tests showed the following results: haemoglobin $151 \mathrm{~g} / \mathrm{l}$; haematocrit 0.424 ; red blood cell count $4.72 \times 10^{12} / 1$; white blood cell $8.8 \times$ $10^{9} / 1$; bilirubin $6 \mathrm{mmol} / \mathrm{l}$; aspartate transaminase $30 \mathrm{IU} / 1$ (normal range $0-18 \mathrm{IU} / 1$ ); alanine transaminase $26 \mathrm{IU} / 1$ (0-22 IU/1); serum iron $36 \mathrm{mmol} / 1$ (13-33 mmol/l); total iron-binding capacity $81 \mathrm{mmol}(41-63 \mathrm{mmol})$; saturation $47 \%$ (25-56\%); ferritin $437 \mu \mathrm{g} / 1(24-250 \mu \mathrm{g} / \mathrm{l})$. There was no history of transfusions, intramuscular injection of iron, haemolysis, chronic illness or alcoholism. Laparoscopy showed multiple well-defined hepatic lesions. Biopsies were taken from three lesions and hepatic parenchyma. HLA typing was performed and revealed HLA Type A3 B7. Blood work performed on family members showed a sibling with the same HLA type, high serum iron, ferritin and saturation index. However, ultrasound of the parents and four siblings revealed no hepatic lesions.
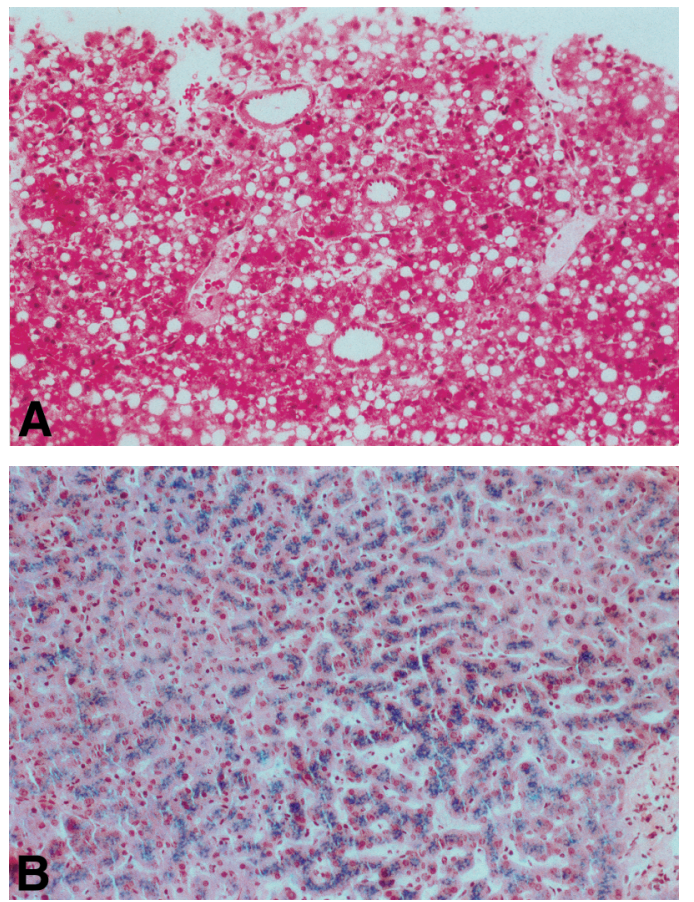

Figure 2 (A) Hepatic adenoma with fatty changes and evident vascularity; (B) adjacent parenchyma shows iron overload
Both the initial and the most current liver biopsy samples showed similar changes. There were lesions clearly distinguishable from the adjacent liver parenchyma. They were composed of three or more cell thick cords of benign appearing hepatocytes with uniform nuclei and pale, vacuolar cytoplasm due to marked fatty changes (figure 2A). Portal tracts were absent, but several medium sized vessels were present within the lesions.

The adjacent normal appearing parenchyma revealed massive deposition of haemosiderin pigments in hepatocytes (grade $3 \& 4$ by the modified Scheuer histologic grading method for iron), ${ }^{5}$ and bile duct cells with mild portal fibrosis and no evidence of cirrhosis (figure 2B). The adenomas were completely devoid of haemosiderin pigments. Hepatic iron assayed on paraffin-fixed tissue was $115.7 \mu \mathrm{mol} / 1$ and hepatic iron index was 4.45 .

\section{Discussion}

Hepatocellular adenomatosis is a very uncommon disease, is characterised by the presence of numerous adenomas (more than four) within an otherwise normal parenchyma. ${ }^{1}$ Since the description of the disease in 1985, 16 cases have been reported in the literature, excluding those present in association with glycogen storage disease, oral contraceptives or androgenic steroids. ${ }^{12}$ It is recognised as an entity distinct from hepatocellular adenoma. ${ }^{6}$ Hepatocellular adenomas are usually single or occasionally multiple, arise in women of child-bearing age, with strong relation to prolonged use of oral contraceptive, anabolic/androgenic steroids, non-contraceptive oestrogen, danazol, virilising and feminising ovarian tumours, type I glycogen disease and diabetes mellitus. ${ }^{1}$ The prevalence of hepatic adenomatosis is equal in men and women. In men, the tumour has been associated with anabolic corticosteroids. ${ }^{7}$ The patient under discussion was asymptomatic until his accident with the diagnosis becoming apparent after a CT scan and a needle liver biopsy, followed by thorough clinical and biochemical examination.

The pathogenesis of hepatocellular adenomatosis remains unknown. This is the first case report of hepatic adenomatosis with primary haemochromatosis, and it is possible that this may be an incidental finding. However, adenomas have been described with iron overload. Review of the world literature reveals two case reports of single hepatic adenomas described in patients with secondary haemochromatosis, complicating thalassaemia. ${ }^{34}$ Genetic haemochromatosis is an autosomal recessive disorder that is associated with HLA-A3, B7 and B14 MHC antigens, and a putative defect on the short arm of chromosome $6 .^{8}$ The precise cytopathological mechanisms by which iron induces neoplastic transformation is unknown, but may be due in part to induction of hepatic damage with resultant hepatocyte regeneration. Iron has been demonstrated to be directly mutagenic, possibly by peroxidative damage to DNA. ${ }^{9}$ The pathogenesis of hepatic adenomatosis in iron overload is not clear. The lack of 


\section{Summary points}

- hepatic adenomatosis is defined by the presence of multiple adenomas of an uncertain aetiology

- the natural history and treatment of liver adenomatosis are not yet well defined hence clinical and radiological follow-up is mandatory, as the risk of malignant changes is not known

- a conservative surgical approach is advocated for symptomatic large lesions

- this entity is rare but should be considered in the differential of multiple hepatic lesions

iron in these lesions is an indication that these adenomas function independently from the rest of the liver and suggest that a neoplastic process is involved in their formation.

This case presents a dilemma as to future management. There is a potential risk of devel-

1 Arsenault TM, Johnson CD, Gorman B, Burgart LJ. Hepatic adenomatosis. Mayo Clin Proc 1996;71:478-80.

2 Propst A, Propst T, Waldenberger P, Vogel W, Judmaier G. A case of hepatocellular adenomatosis with a follow-up of 11 years. Am $\mathcal{F}$ Gastroenterol 1995; 90:1345-6.

3 Shuangshoti S, Thaicharoen A. Hepatocellular adenoma in a beta-thalassaemia woman having secondary iron overload. f Med Assoc Thai 1994;77:108-12.

4 Cannon RO, Duskeiko, GM, Long JA, et al. Hepatocellular adenoma in a young woman with B-thalassaemia and secondary iron overload. Gastroenterology 1981;81:352-5.

5 Sheuer PJ, William R, Muir AR. Hepatic pathology in relatives of patients with haemochromatosis. F Pathol 1962;84: tives of

6 Flejou JR, Barge J, Menu Y, et al. Liver adenomatosis an entity distinct from liver adenoma. Gastroenterology 1985;89 $1132-8$. oping cirrhosis and hepatocellular carcinoma in a patient with haemochromatosis. Intratumoural bleeding causing abdominal pain and haemoperitoneum has been reported in adenomatosis. ${ }^{1}$ Therapeutic manoeuvres include removal of iron, monitoring of liver function tests and radiological follow-up to determine if the lesions change. The patient is young and otherwise healthy and needs to be considered for hepatic transplantation should cirrhosis or malignant transformation occur. However, the outcome of liver transplantation in patients with haemochromatosis is rather poor. Survival of patients with haemochromatosis after transplantation is decreased compared to other recipients, due to cardiac, infectious and malignant complications which account for excess morbidity and mortality in these patients. ${ }^{9}$ A conservative surgical approach with resection of large or symptomatic lesions and observation of smaller lesions has been advocated. ${ }^{10}$

7 Boyd PR, Mark GJ. Multiple hepatic adenomas and a hepatocellular carcinoma in a man on oral methyl testosterone for eleven years. Cancer 1997;40:1765-70.

8 Witte OL, Crosby WH, Edwards CQ, Fairbanks VF, Mitros FA. Hereditary haemochromatosis. Clin Chem Acta 1996; 245:139-200.

9 Loeb LA, James EA, Waltersdorph AM, Kelbanoff SJ. Mutagenesis by the autooxidation of iron with isolated DNA. Proc Natl Acad Sci USA 1988;85:3918-22.

10 Ribeiro A, Burgart LJ, Nagorney DM, Gores GJ. Management of liver adenomatosis: results with a conservative surgical approach. Liver Transpl Surg 1998;4:388-98. 


\title{
An aggressive intrasinusoidal lymphoma presenting with marked systemic disturbance but normal imaging studies
}

\author{
Ross Camidge, Edmund Ong, Helen Lucraft
}

\begin{abstract}
Summary
A patient with an aggressive intrasinusoidal non-Hodgkins lymphoma, presenting with marked systemic disturbance but only a mildly raised alkaline phosphatase as a localising sign is described. All imaging studies of the liver were normal and the diagnosis was delayed until a percutaneous liver biopsy was performed. Once diagnosed, the patient responded extremely well to conventional antilymphoma chemotherapy.
\end{abstract}

Keywords: lymphoma; fever of unknown origin

Mildly abnormal liver function tests detected during the investigation of a pyrexia of unknown origin (PUO) are often disregarded in the presence of normal imaging studies of the liver. However, such results are not always epiphenomena. Similarly, the finding of a normal appearing hepatic parenchyma on ultrasound or computed tomography (CT) scanning does not necessarily exclude significant pathology. Here we report the case of an aggressive intrasinusoidal lymphoma of the liver, presenting as a PUO, with only a mildly raised alkaline phosphatase as an initial localising feature.

\section{Case report}

A previously well 62-year-old Caucasian man was referred to the Infectious Diseases Unit, Newcastle General Hospital, with a PUO. He reported 3 months of general malaise, recurrent drenching sweats, rigors and $15 \mathrm{~kg}$ weight loss. Apart from evidence of recent weight loss and temperatures spiking up to $39^{\circ} \mathrm{C}$ several times a day, there were no other abnormalities on physical examination.

Autoimmune and extensive microbiological investigations including serology for HIV, hepatitis $\mathrm{A}, \mathrm{B}$ and $\mathrm{C}$ were negative. A bone marrow biopsy showed only reactive plasma cells. Cerebrospinal fluid analysis including cytology was unremarkable. A bone scan, an abdominal ultrasound scan and a CT scan of the thorax and abdomen all appeared normal.

Inflammatory markers on admission revealed a normal white cell count of $4.9 \times 10^{9} / 1$ (normal differential) but an erythrocyte sedimentation rate of $137 \mathrm{~mm} / \mathrm{h}$ and a C-reactive protein of $347 \mathrm{mg} / 1$ (normal < 10). Immuno- globulin levels and clotting times were unremarkable. Liver function tests showed a low albumin at $25 \mathrm{~g} / 1$ (normal 34-50) with normal levels of bilirubin, alanine transaminase and gamma-glutamyl transferase. A mildly raised alkaline phosphatase at $169 \mathrm{U} / 1$ (normal 65-100) was felt to be 'reactive'.

The patient deteriorated rapidly, losing weight, becoming extremely weak and intermittently confused. He developed oral ulceration, a normochromic, normocytic anaemia (haemoglobin $8.1 \mathrm{~g} / \mathrm{dl}$ ) and thrombocytopenia $\left(26 \times 10^{9} / 1\right)$. By the third week of his hospital admission the patient had become moribund. His alkaline phosphatase had risen to $293 \mathrm{U} / 1$ and his lactate dehydrogenase to $1025 \mathrm{U} / 1$ (normal < 430).

Following platelet infusion pre-procedure, a percutaneous liver biopsy revealed an intrasinusoidal high-grade centroblastic B-cell nonHodgkins lymphoma (figure 1). With the already available imaging information this was designated stage $1 \mathrm{E}$ non-Hodgkins lymphoma with B symptoms.

Initial therapy with high-dose intravenous (iv) dexamethasone (4 mg qid) produced a dramatic fall in temperature (figure 2). Urgent combination chemotherapy was then commenced using 3-week cycles of CHOP (cyclophosphamide $750 \mathrm{mg} / \mathrm{m}^{2}$ iv, adriamycin 50 $\mathrm{mg} / \mathrm{m}^{2}$ iv, vincristine $2 \mathrm{mg}$ iv, with oral prednisolone $40 \mathrm{mg}$ daily for 5 days). A reduced dose $(75 \%)$ was used in the first course and full doses thereafter. By the sixth

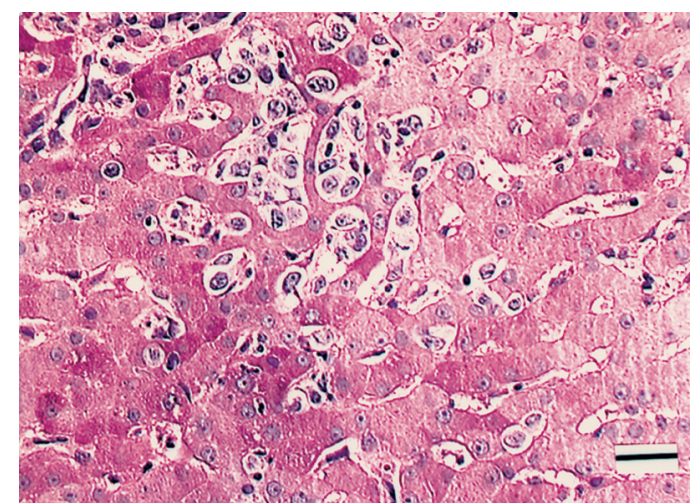

Figure 1 Liver biopsy (periodic acid-Schiff stain). Note presence of abundant large pleomorphic lymphoid cells in the hepatic sinusoids. Immunostaining showed the cells to be CD20 and CD 45 positive. A $25-\mu \mathrm{m}$ scale marker is shown 


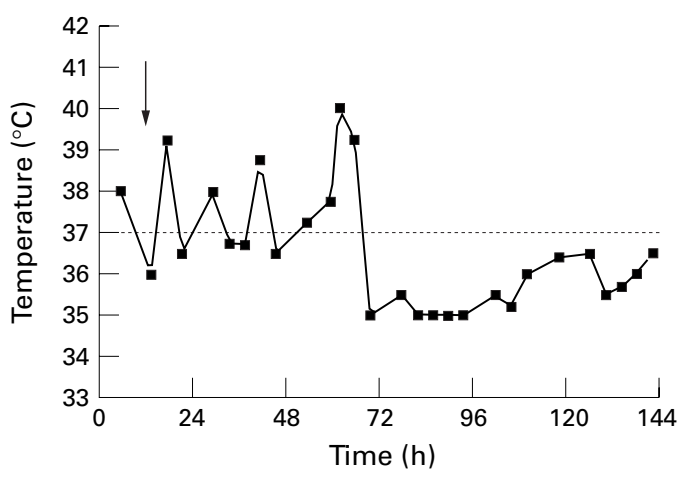

Figure 2 Temperature chart showing lysis of patient's pyrexia following commencement of high dose dexamethasone (arrow). Intervals of 24 hours (at midnight) are marked

and final course he was apyrexial, had gained $18 \mathrm{~kg}$ in weight and his liver function tests had normalised. A repeat liver biopsy performed one month after his last course of chemotherapy showed no evidence of persistent lymphoma. Six months later he remains clinically and biochemically disease-free. A CT scan of the abdomen, pelvis and thorax performed during follow-up appeared normal, as had all previous imaging modalities.

\section{Discussion}

Primary non-Hodgkins lymphoma of the liver (stage 1E) is rare. ${ }^{1}$ A $4: 1$ male to female preponderance with a mean age of onset in the 5 th decade has been described. ${ }^{2}$ In $50 \%$ of cases B symptoms, particularly weight loss, appear prominent. Biochemically, $80 \%$ of cases show an elevated alkaline phosphatase, and $100 \%$ show an elevated lactate dehydrogenase. $^{34}$ The majority are diffuse

1 Lei K, Chow J, Johnson P. Aggressive primary hepatic lymphoma in Chinese patients. Cancer 1995;76:1336-43.

2 Anthony P, Sarsfield P, Clarke T. Primary lymphoma of the liver: clinical and pathological features of 10 patients. $f$ Clin Pathol 1990;43:1007-13.

3 Aozasa K, Mishima K, Ohsawa M. Primary malignant lymphoma of the liver. Leukemia Lymphoma 1993;10:353-7.

\section{Learning points}

- primary lymphoma of the liver can present with severe systemic disturbance but few localising signs

- normal ultrasound or CT imaging studies of the liver do not exclude significant hepatic pathology

- mild elevation of alkaline phosphatase levels occurring during PUO is not always nonspecific

large cell lymphomas of B-cell lineage. ${ }^{3}$ The prognosis with treatment appears variable, ranging from $14 \%$ to $56 \%$ survival 4 years after diagnosis. ${ }^{12}$ In the present case, thrombocytopenia was a prominent feature, previously noted commonly only in Chinese patients. ${ }^{1}$ The lack of hepatomegaly was unusual, being present in the majority $(63-100 \%)$ of previously reported cases. ${ }^{1-5}$ The unique feature in this patient was the normal appearance of the liver on ultrasound and CT scanning. In all other recorded cases, imaging studies of the liver have shown either a diffuse abnormality or a uni-/multi-nodular pattern, ${ }^{1-5}$ directly precipitating biopsy.

This case demonstrates that mildly abnormal liver function tests in a patient with a PUO should not necessarily be dismissed as nonspecific findings. The case also demonstrates that a normal liver ultrasound or CT scan in this situation does not exclude relevant hepatic pathology. Percutaneous liver biopsy proved to be diagnostic. We recommend a low threshold for liver biopsy in the investigation of PUOs in a similar clinical setting, having ensured an adequate platelet count and clotting beforehand.

4 Osborne B, Butler J, Guarda L. Primary lymphoma of the liver: ten cases and a review of the literature. Cancer 1985;56:2902-10.

5 Dement S, Mann R, Staal S, et al. Primary lymphomas of the liver: report of six cases and review of the literature. $A m$ f Clin Pathol 1987;88:255-63. 


\title{
Aspergillus niger endocarditis in an immunocompetent patient: an unusual course
}

\author{
Yitshak Kreiss, Zvi Vered, Nathan Keller, Ilan Kochva, Yechezkel Sidi, Hanan Gur
}

\begin{abstract}
Summary
Aspergillus is an opportunistic nosocomial fungus generally associated with a high mortality rate. $A$ niger has been rarely associated with infection, and most cases have occurred in patients who have recently undergone heart surgery or in immunocompromised patients. We present a case of an immunocompetent patient with $A$ niger endocarditis which illustrates the difficulties in diagnosis and the possible insidious course of fungal endocarditis.
\end{abstract}

Keywords: endocarditis; Aspergillus niger; transoesophageal echocardiography

Aspergillus is an opportunistic nosocomial fungus generally associated with a high mortality rate. ${ }^{1}$ While $A$ fumigatus has been reported to be the most frequent cause of aspergillosis, $A$ niger has been rarely associated with infection, and most cases have occurred in patients who have recently undergone heart surgery for valvular replacement and pacemaker implantation, as well as in patients with neoplastic diseases, intravenous treatment or drug addiction, long-term parental feeding and immunosuppression. ${ }^{2}$

The optimal therapy for Aspergillus endocarditis has not been established, but it usually requires combined aggressive medical and surgical treatment, ${ }^{3}$ as high-dose amphotericin alone is ineffective because of the resistance of many Aspergillus species and poor penetration into tissues, while surgical treatment alone may be insufficient, as most patients have disseminated disease at the time of diagnosis. ${ }^{3}$ The length of therapy, the efficacy of alternative modalities, such as liposomal amphotericin or new imidazole derivatives, are of unproved value as yet. ${ }^{4}$

We present a case of an immunocompetent patient with $A$ niger endocarditis which illustrates the difficulties in diagnosis and the important role of repeated transoesophageal echocardiography (TOE) in proper management of infective endocarditis.

\section{Case report}

A 57-year-old man was admitted for malaise and fever of 5 days duration, 2 months after mitral valve repair for severe mitral valve prolapse and regurgitation. Apart from fever $\left(38.5^{\circ} \mathrm{C}\right)$ and an apical systolic murmur, his physical examination was unremarkable. His blood pressure was $110 / 70 \mathrm{mmHg}$, the pulse rate 96 beats $/ \mathrm{min}$, and there were no signs of petechiae, splenomegaly, clubbing, or other signs of endocarditis. The chest $\mathrm{X}$-ray and electrocardiogram were within normal limits. Laboratory results showed accelerated erythrocyte sedimentation rate (ESR $120 \mathrm{~mm} / \mathrm{h}$, Westergren) and mild normocytic, normochromic anaemia (haematocrit ratio 0.33 ). The urinalysis was normal, without haematuria, as were the results of serum biochemical profile. Seven blood culture sets were negative, as were serology for rickettsia and $\mathrm{Q}$ fever. At that time we performed TOE, which revealed a $4 \mathrm{~mm}$ mobile lesion on the mitral valve, suggestive of vegetation, but without evidence for valvular damage. With the diagnosis of culture-negative prosthetic valve endocarditis, vancomycin and gentamycin were administered and the fever subsided. After 2 weeks, low-grade fever reappeared, and the treatment was changed to vancomycin, ciprofloxacin and rifampin. After 6 weeks of parenteral antibiotic treatment the patient's general condition improved markedly, the fever and anaemia resolved and the elevated ESR decreased, and the patient, who was apparently cured, was ready to be discharged. However, to our surprise, a routine predischarge TOE demonstrated a marked increase in the vegetation to $11 \mathrm{~mm}$, with a highly mobile appearance (figure 1). The patient was then referred for surgery, and a large friable vegetation on the mitral valve was found and excised.

Direct microscopy of the vegetation stained with $10 \% \mathrm{KOH}-Q$ uink revealed septate hyphae and culture grew $A$ niger (figure 2). The patient had been treated with amphotericin for two

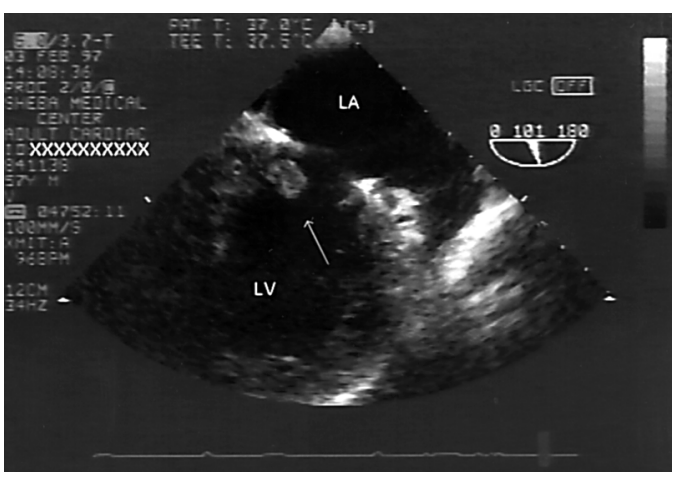

Figure 1 TOE after 6 weeks of antibiotic treatment showing the $11 \mathrm{~mm}$ vegetation (arrow). At that time the patient was afebrile, ready to be discharged 


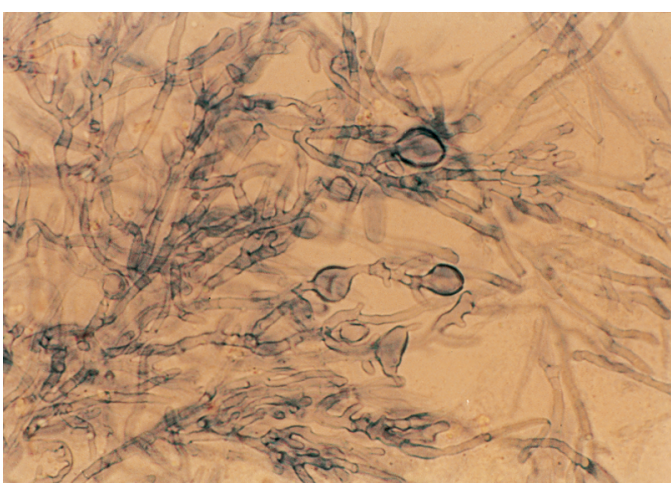

Figure $2 \mathrm{KOH}-Q u i n k$ stain of the vegetation showing septate hyphae typical of Aspergillus sp later defined as $A$ niger. Orig $\times 40$

months and subsequently with itraconazole for a year. A repeat TOE 6 months after surgery revealed only mild mitral regurgitation. At present, 16 months after surgery, the patient is doing well, without evidence of recurrence.

\section{Discussion}

The present case illustrates the difficulties in diagnosis and the possible insidious course of fungal endocarditis. The only predisposing factor for $A$ niger infection in this patient was the open heart surgery. Only one previous report of a surviving case of $A$ niger endocarditis has recently been reported. ${ }^{5}$ As in the

Andriole VT. Infections with Aspergillus species. Clin Infect Dis 1993; 17:5481-6.

Stavridis GT, Shbbo FP. Aspergillus prosthetic valve endocarditis. Eur f Cardiothorac Surg 1993;7:50-1.

3 Denning DW, Stevens DA. Antifungal and surgical treatment of invasive aspergillosis: a review of 2121 published cases. Rev Infect Dis 1990;12:1147-201. present case, the patient was immunocompetent. However, the present case is distinct for the 'benign' course and the apparent 'clinical cure' after 6 weeks of antibacterial treatment. The TOE findings were fundamental both in diagnosis and in our decision to refer the patient to immediate surgery. Indeed, TOE, as demonstrated in the present case, is a powerful diagnostic method, with an established accuracy for vegetations of over $90 \% .^{6}$ Although stratification of patients into groups that are at high risk for systemic emboli and death according to vegetation size is still controversial, data from echocardiographic studies suggest that patients with vegetations $>10 \mathrm{~mm}$ in diameter are at increased risk of embolic complications, particularly when it involves the mitral valve or when it has a high grade of mobility. ${ }^{6}$ Moreover, serial TOE examinations should increase the specificity and diagnostic accuracy of the findings and provide stronger instrument for decision making. In our patient, the vegetation size, its mobility and the involvement of the mitral valve, were all criteria which led us to refer the patient for surgery despite his excellent clinical condition.

In conclusion, we presented a case of $A$ niger endocarditis who had had an insidious course and a favourable outcome of surgery combined with prolonged antifungal treatment. The case emphasizes the role of serial TOE in the diagnosis and management of patients with infective endocarditis, particularly in patients with culture-negative endocarditis.

4 Hosking MC, Macdonald NE, Cornel G. Liposoma mphotericin B for postoperative Aspergillus fumigatus endocarditis. Ann Thorac Surg 1995;59:1015-7.

5 Vivas C. Endocarditis caused by Aspergillus niger: case report. Clin Infect Dis 1998;27:1322-3.

6 Daniel WG, Mugge A. Transesophageal echocardiography. N Engl f Med 1995;332:1268-79. 